\title{
Identification of a novel gene (Apq1) from the indica rice cultivar 'Habataki' that improves the quality of grains produced under high temperature stress
}

\author{
Kazumasa Murata $^{\dagger 1)}$, Yukihide Iyama ${ }^{1)}$, Takuya Yamaguchi ${ }^{1)}$, Hidenobu Ozaki ${ }^{1,2)}$, Yoshinori Kidani ${ }^{1,3)}$ \\ and Takeshi Ebitani ${ }^{\dagger * 1)}$ \\ 1) Toyama Prefectural Agricultural, Forestry \& Fisheries Research Center, 1124-1 Yoshioka, Toyama, Toyama 939-8153, Japan \\ 2) Present address: Toyama Prefectural Agricultural Food Product Division, 1-7 Shinsogawa, Toyama, Toyama 930-8501, Japan \\ 3) Present address: Toyama Agriculture and Forestry Promotion Center, 1-3-22 Suwanogawara, Toyama, Toyama 930-0088, Japan
}

\begin{abstract}
The appearance of brown rice grown under high temperature conditions is an important characteristic for improvement in Japanese rice breeding programs. We performed a QTL analysis of the appearance quality of brown rice using chromosome segment substitution lines of the indica cultivar 'Habataki' in the 'Koshihikari' genetic background. A line carrying a 'Habataki' segment on chromosome 7 showed a high percentage of perfect grains produced under high temperature conditions during the ripening period. To verify the role of this segment, and to narrow down the region containing the useful allele, substitution mapping was performed using multiple paired lines. As a result, the chromosomal location of a gene that we named Appearance quality of brown rice 1 (Apql) was delimited to a 48-kb region. In addition, we developed an Apql-near isogenic line (NIL) to evaluate the effect of $A p q 1$ on various agronomic traits. Under high temperature conditions during the ripening period, the Apq1-NIL produced significantly higher percentages of perfect grains than 'Koshihikari'. Other agronomic traits, including yield and palatability, were similar between the Apq1-NIL and 'Koshihikari'. Therefore, the 'Habataki' allele of $A p q 1$ will be useful in breeding programs aimed at improving the quality of grains ripened under high temperature conditions.
\end{abstract}

Key Words: Apq1, appearance quality, grain, 'Habataki', 'Koshihikari’, mapping, rice (Oryza sativa L.).

\section{Introduction}

Recently in Japan, high temperatures during the ripening period have resulted in deterioration in the quality of rice grains. This deterioration is due to increases in the abundance of chalky grains, which decrease milling quality. The price of brown rice is fixed by grain appearance, particularly the ratio of chalky grains. Therefore, the development of novel cultivars with tolerance to high temperature stress during the ripening period is a major objective in breeding programs. In particular, improvements of this trait in 'Koshihikari', which is a leading cultivar in Japan, are strongly needed (http://www.maff.go.jp/j/seisan/kankyo/ ondanka/pdf/h22_tekiou_gijyutu_gaiyo.pdf).

The popularity of 'Koshihikari' is due to its good palatability. The development of near isogenic lines (NILs) with desirable traits in the 'Koshihikari' genetic background is a practical approach to developing new cultivars with good eating quality. Indeed, NILs for several traits, including blast resistance, early and late heading, and short culms, have already been developed using 'Koshihikari' as the ge-

Communicated by M. Yano

Received March 12, 2014. Accepted July 29, 2014.

*Corresponding author (e-mail: takeshi.ebitani@pref.toyama.lg.jp)

${ }^{\dagger}$ These authors contributed equally to this work netic background (Ebitani et al. 2011, Fukuoka et al. 2009, Ishizaki et al. 2005, Kojima et al. 2004, Takeuchi et al. 2006, Wan et al. 2005a).

The use of DNA marker-assisted selection (MAS) is highly effective in developing NILs (Ebitani et al. 2011, Fukuoka et al. 2009, Takeuchi et al. 2006, Wan et al. 2005a). To facilitate the development of lines with tolerance to high temperatures, it is required to identify quantitative trait loci (QTLs) or genes that improve the appearance quality of brown rice under high temperature stress. Previously, Ebitani et al. (2008) detected four QTLs in the indica cultivar 'Kasalath' that increased the percentage of perfect grains (PPG) in plants with a 'Koshihikari' genetic background. The 'Kasalath' allele of the locus on chromosome 12 that had the highest effect among those QTLs was effective at approximately $27^{\circ} \mathrm{C}$, the average air temperature during the 20 days after heading (hereafter referred to as the 'average temperature'), but not at $28^{\circ} \mathrm{C}$ or higher (Ebitani et al. 2013). Kobayashi et al. (2013) reported that the $q W B 6$ gene derived from the tolerant cultivar 'Hana-echizen' (HE) could decrease the abundance of white-back kernels at approximately $28^{\circ} \mathrm{C}$ in the 'Niigata-wase' genetic background, and could reduce heat-induced quality decline to the same level as in HE. However, it remains unclear whether the HE allele at $q W B 6$ would be effective in the 'Koshihikari' genetic background. Tabata et al. (2007) detected three QTLs 
in the heat-tolerant cultivar 'Koshiji-wase' that decreased the incidence of white-back kernels at daily mean temperatures ranging from $25.0^{\circ} \mathrm{C}$ to $30.7^{\circ} \mathrm{C}$. These QTLs have not been mapped precisely yet.

In the present study, we sought to identify a gene in the indica cultivar 'Habataki' that confers tolerance to high temperature stress during the ripening period. We focused on 'Habataki' because it produces very few white-back and basal-white kernels, which are the main causes of grain quality reduction under high temperature stress. This is despite the fact that the appearance quality of 'Habataki' itself is poor due to the frequent occurrence of milky-white kernels and white-berry kernels (Supplemental Fig. 1 and Kobayashi et al. 1990). Chromosome segment substitution lines (CSSLs) of 'Habataki' in the 'Koshihikari' genetic background were developed as experimental material for QTL analysis. We identified and mapped a QTL and named it Apq1 (Appearance quality of brown rice 1). Furthermore, we developed a NIL containing the 'Habataki' Apq1 allele in the 'Koshihikari' genetic background. The effect of this 'Habataki' allele at Apql was evaluated by assessing the responses of the NIL plants to high temperature stresses in air temperature-controlled growth chambers as well as under field conditions. Various agronomic traits were also assessed in the NIL to evaluate the suitability of this line for practical use in paddy fields.

\section{Materials and Methods}

Plant materials, growth conditions, and assessment of brown rice appearance quality

Thirty-two CSSLs (hereafter referred to as 'KHSLs') were developed through a process illustrated in Supplemental Fig. 2. For QTL detection and mapping, 'Habataki', 'Koshihikari', the KHSLs, and paired line populations (fixed lines derived from recombinants in QTL candidate chromosomal regions; see Results for details) were transplanted in late April at distances of $25 \mathrm{~cm}$ between plants and $25 \mathrm{~cm}$ between rows in a paddy field at the Toyama Agricultural Research Institute (TARI) in Toyama, Japan $\left(36.4^{\circ} \mathrm{N}, 137.1^{\circ} \mathrm{E}\right)$. Nitrogen fertilizer was applied at customary rates (basal dressing $0.8 \mathrm{~kg} / \mathrm{a}$; top dressing $0.3 \mathrm{~kg} / \mathrm{a}$ ). At maturity, 10 plants of each line were harvested in bulk and threshed. After de-husking, immature grains were removed. Because of differences in grain thickness among the KHSLs, a mill-grade sieve with a mesh size of $1.7 \mathrm{~mm}$ was used in QTL detection. For QTL mapping, a 1.9-mm sieve was used. One hundred grains were randomly selected from each line and were classified as perfect grains, chalky grains, or other grains by visual inspection. Perfect grains were defined as non-chalky with a normal shape. Grains with unusual shapes or that were stained were classified as 'other' grains. Chalky grains were sub-classified as basalwhite kernels, white-back kernels, white-berry kernels, milky-white kernels, or white-core kernels, as described by Ebitani et al. (2008). When two of these sub-classifications occurred simultaneously (for example, basal-white and white-berry kernels), the grains were classified based on the following list, in order of priority: milky-white, white-back, basal-white, white-berry, and white-core.

\section{QTL detection and mapping}

QTL detection using the KHSLs was performed in 2008, and QTL mapping using paired line populations was performed in 2010, 2012, and 2013. For QTL detection, the PPG of each line (including 'Habataki') was compared to that of 'Koshihikari', with three replications. The heading date and 1,000-grain weight were also evaluated since these traits are related to the appearance quality of brown rice (Hori et al. 2012 for heading date; Ebitani et al. 2008 for 1,000-grain weight). The differences between 'Habataki', the KHSLs, and 'Koshihikari' were statistically evaluated using Dunnett's multiple comparison test. For QTL mapping, $t$-tests were performed to compare PPG values between KHSL-18 and KHSL-19 or between the 'Habataki' and 'Koshihikari' homozygous lines in paired line populations, with four replications. When there were significant differences in PPGs between two lines, we hypothesized that a QTL was present in the chromosomal region that differed between the lines. A probability level of 0.05 was used as the threshold for the detection of putative QTLs.

\section{DNA marker analysis}

RFLP marker analyses were performed according to the method of Kurata et al. (1994), using the ECL Direct Nucleic Acid Labeling and Detection System (Amersham Pharmacia, Uppsala, Sweden). Total plant DNA was extracted by the CTAB method from leaves (Murray and Thompson 1980). SSR marker analyses were performed as follows. Each PCR mixture contained $10 \mathrm{ng}$ template DNA, $1.0 \mu \mathrm{L}$ of $10 \times$ PCR buffer (Hokkaido System Science, Hokkaido, Japan), $1.5 \mu \mathrm{L}$ of $2.5 \mathrm{mM}$ of each deoxynucleotide triphosphate (Hokkaido System Science), $1.5 \mu \mathrm{L}$ of $5 \times$ Tuning buffer (Hokkaido System Science), $0.1 \mu \mathrm{L}$ of 2.5 units Taq DNA polymerase (Hokkaido System Science), and $0.5 \mu \mathrm{L}$ of $20 \mathrm{pM}$ of each primer in a total volume of $10.0 \mu \mathrm{L}$. The amplification conditions were as follows: 37 cycles of $95^{\circ} \mathrm{C}$ $(20 \mathrm{~s}), 55^{\circ} \mathrm{C}(1 \mathrm{~min})$, and $72^{\circ} \mathrm{C}(30 \mathrm{~s})$, with a final extension at $72^{\circ} \mathrm{C}$ for $5 \mathrm{~min}$. The primer sequences for the SSR markers shown in Supplemental Figs. 3, 4 and Fig. 4 (see Results) were obtained using data from McCouch et al. (2002) and the International Rice Genome Sequencing Project (2005), respectively. The markers EB_1, EB_2, EB_3, EB_ Indel-03, EB Indel-05, Tak6166-3, and Tak6168-8 were designed for the present study, and markers RM21975-2, RM21977-2, and RM21980-2 were modified for use in the present study (Supplemental Table 1). For analysis, the amplified DNA products were separated by electrophoresis on $3 \%$ agarose gels. 
Evaluation of agronomic traits and grain appearance quality under high temperature stress of the Apq1-NIL

We used two lines, the Apql-NIL and a control NIL (cont-NIL), to evaluate the usefulness of the 'Habataki' Apq1 allele. These two lines were used in QTL mapping. The Apq1-NIL had a relatively small substituted 'Habataki' segment while the cont-NIL lacked this segment.

The Apq1-NIL, the cont-NIL, and 'Koshihikari' plants were transferred at the time of maximum tillering from a paddy field to $1 / 5000$ a Wagner pots (one plant per pot), and the pots were placed in a water pool under natural conditions. When the first panicle in each pot had emerged, 10 pots per line were transferred to two growth chambers (five plants per line in each chamber). Nitrogen fertilizer was applied at $0.3 \mathrm{~g} /$ pot at the young panicle formation stage. The growth chambers were maintained under natural light conditions and two different temperature regimes (high and normal). The experiments were conducted in 2012 and 2013. In 2012, the high temperature conditions during the ripening period (9 A.M. to 6 P.M./6 P.M. to 9 A.M./average) were $30.5^{\circ} \mathrm{C} / 25.6^{\circ} \mathrm{C} / 27.4^{\circ} \mathrm{C}$, respectively, and the normal temperature conditions were $28.3^{\circ} \mathrm{C} / 23.6^{\circ} \mathrm{C} / 25.4^{\circ} \mathrm{C}$, respectively. In 2013 , the high temperature conditions $\left(31.3^{\circ} \mathrm{C} /\right.$ $26.3^{\circ} \mathrm{C} / 28.2^{\circ} \mathrm{C}$ ) were higher than in 2012 , while the normal temperature conditions $\left(28.6^{\circ} \mathrm{C} / 23.4^{\circ} \mathrm{C} / 25.3^{\circ} \mathrm{C}\right)$ were similar to those in 2012. The temperatures of the water pools in both growth chambers were kept constant at $25^{\circ} \mathrm{C}$. At maturity, all kernels in each pot were harvested and the appearance quality was evaluated as described above.

In 2011 and 2012, the Apq1-NIL and 'Koshihikari' were cultivated in a paddy field at TARI. We raised 160 plants per plot, with three replications at a planting density of $22.9 \mathrm{hills} / \mathrm{m}^{2}$. Nitrogen fertilizer was applied at customary rates (basal dressing $0.8 \mathrm{~kg} / \mathrm{a}$; top dressing $0.3 \mathrm{~kg} / \mathrm{a}$ ). Various agronomic traits, including heading date, culm length, panicle length, number of panicles, yield, and palatability, were evaluated using the method of Yamamoto et al. (1996).

\section{Results}

\section{Development of KHSLs}

The procedure used to develop the 32 KHSLs is illustrated in Supplemental Fig. 2. 'Koshihikari' (as the female) was crossed with 'Habataki', and the resultant $\mathrm{F}_{1}$ plants were backcrossed to 'Koshihikari' to produce $81 \mathrm{BC}_{1} \mathrm{~F}_{1}$ plants. All of these $\mathrm{BC}_{1} \mathrm{~F}_{1}$ plants were again backcrossed to 'Koshihikari' to produce $\mathrm{BC}_{2} \mathrm{~F}_{1}$ seeds. The $\mathrm{BC}_{2} \mathrm{~F}_{1}$ plants (one from each backcross) were analyzed using 77 DNA markers distributed over the whole genome (Supplemental Fig. 3). Based on this whole-genome survey, we used foreground selection to select target chromosomes that were heterozygous. The selected $\mathrm{BC}_{2} \mathrm{~F}_{1}$ plants were again backcrossed to 'Koshihikari' to produce $\mathrm{BC}_{3} \mathrm{~F}_{1}$ seeds. We then used a whole-genome survey with both foreground and background selection to minimize the occurrence of heterozygotes in non-target chromosomal regions. As a result, 63
$\mathrm{BC}_{3} \mathrm{~F}_{1}$ plants were selected. One plant was heterozygous only in its target region and was homozygous for 'Koshihikari' in all other regions; therefore, this plant was selfpollinated and its $\mathrm{BC}_{3} \mathrm{~F}_{2}$ seeds were harvested. The other $62 \mathrm{BC}_{3} \mathrm{~F}_{1}$ plants were backcrossed to 'Koshihikari' one or two more times. Background selection was performed in the $\mathrm{BC}_{4} \mathrm{~F}_{1}$ and $\mathrm{BC}_{5} \mathrm{~F}_{1}$ generations. Plants that had a 'Habataki' homozygous segment in their target regions (candidates for KHSLs) were selected from among the $\mathrm{BC}_{3} \mathrm{~F}_{2}, \mathrm{BC}_{4} \mathrm{~F}_{2}$, and $\mathrm{BC}_{5} \mathrm{~F}_{2}$ populations. If the selected plants had heterozygous segments in non-target regions, background selection was again performed in the following generations. Finally, the whole genomes of 32 plants were examined using 133 SSR markers (Supplemental Fig. 4).

The genotypes of the 32 KHSLs are represented graphically in Fig. 1. The substituted chromosome segments cover most of the 12 chromosomes. However, one region (between RM7279 and RM1359) may not be covered because the substitution segments of KHSL-8 and KHSL-9 do not overlap. In general, each line has one substituted 'Habataki' segment in the 'Koshihikari' genetic background. KHSL-12 contains a small additional 'Habataki' segment (at RM4584 on chromosome 7). If it is assumed that each recombination occurred midway between the two surrounding markers, and based on the physical map of the International Rice Genome Sequencing Project (2005), the average length of the major 'Habataki' chromosome segment in each line should be $18.6 \mathrm{Mbp}$. The percentages of substituted segments on particular chromosomes ranged from $15.9 \%$ to $99.9 \%$, and each chromosome was covered by two to four lines (Supplemental Fig. 4).

\section{Detection of chromosomal region affecting PPGs}

The PPGs of the parental lines 'Habataki' and 'Koshihikari' and of the 32 KHSLs were measured. The PPGs of 'Habataki' and 'Koshihikari' were $18.5 \%$ and $60.0 \%$, respectively (Fig. 2A, 2B). The inferior grain quality of 'Habataki' was due to increased rates of white-berry kernels (39.0\%), white-core kernels $(9.5 \%)$, and milky-white kernels $(31.0 \%)$. Very few white-back or basal-white kernels were found in 'Habataki'. The PPGs of KHSL-5, KHSL-6, KHSL-7, KHSL-19, KHSL-24, and KHSL-32 were significantly higher than that of 'Koshihikari' (Fig. 2A). KHSL-2, KHSL-8, and KHSL-20 had significantly lower PPGs than that of 'Koshihikari' (Fig. 2A). The heading dates of KHSL$5(8 / 13)$ and KHSL-7 (8/16) were much later than that of 'Koshihikari' (7/28), and therefore the average temperatures for these lines $\left(25.0^{\circ} \mathrm{C}\right.$ for KHSL-5 and $24.5^{\circ} \mathrm{C}$ for KHSL7) were much lower than that of 'Koshihikari' $\left(27.4^{\circ} \mathrm{C}\right)$ (Supplemental Table 2). Moreover, many sterile spikelets developed on KHSL-5 plants (data not shown). The 1,000-grain weights of KHSL-5 (18.5 g), KHSL-6 (21.3 g), KHSL-7 (20.8 g), KHSL-24 (20.7 g), and KHSL-32 ( $20.4 \mathrm{~g}$ ), were more than $1 \mathrm{~g}$ lighter than that of 'Koshihikari' (22.7 g) (Supplemental Table 2). On the other hand, the heading date $(8 / 2)$ and 1,000-grain weight $(23.0 \mathrm{~g})$ of 


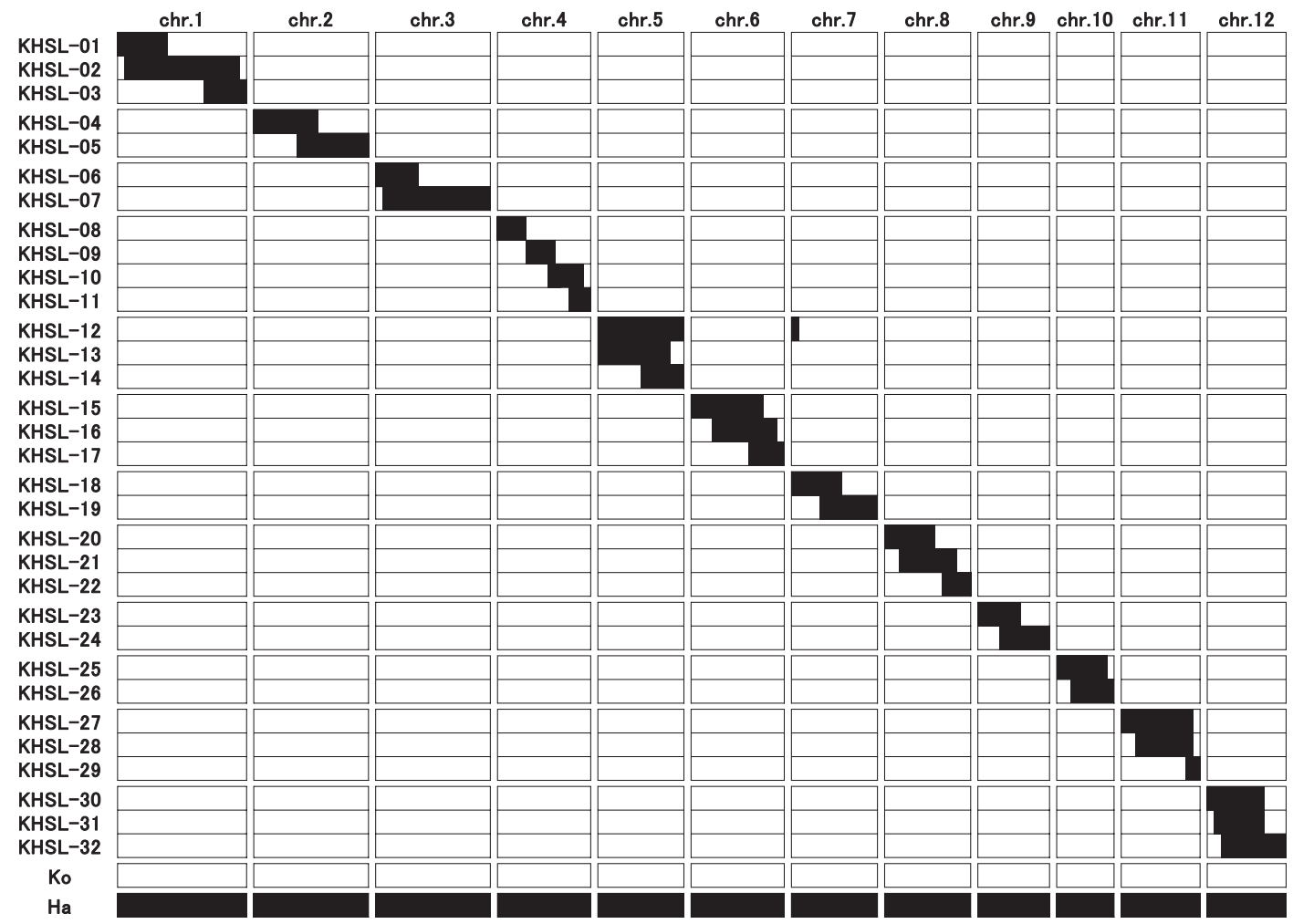

Fig. 1. Graphical representation of genotypes of the 32 KHSLs. Black and white segments indicate regions homozygous for 'Habataki' (Ha) and 'Koshihikari' (Ko), respectively. The genotypes were determined using 133 SSR markers (Supplemental Fig. 4)
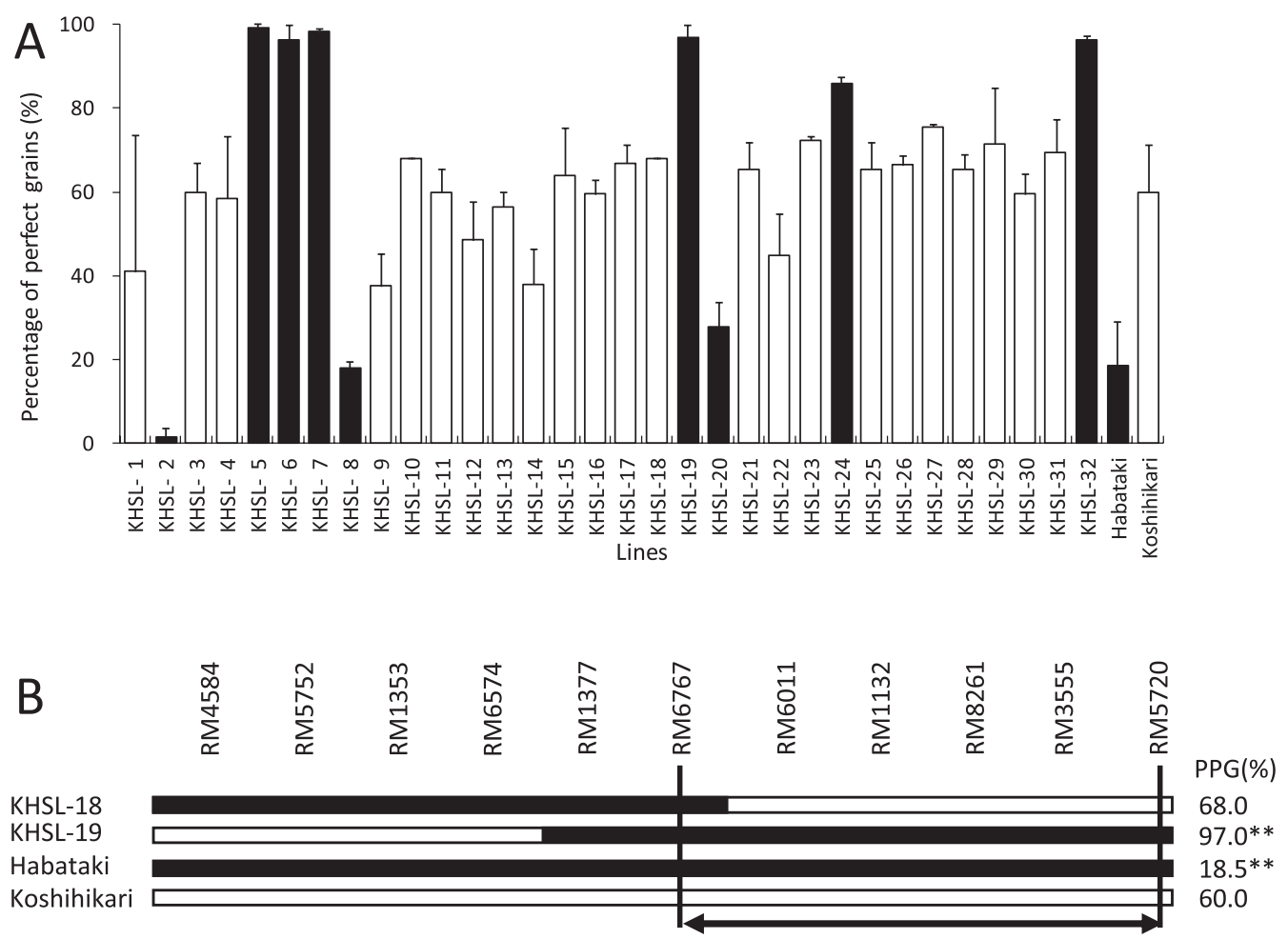

Fig. 2. Comparison of the PPGs of the 32 KHSLs and the parental lines 'Habataki' and 'Koshihikari'. A. Black bars indicate significant differences between 'Habataki' or the KHSL and 'Koshihikari' at $P<0.05$, according to Dunnett's multiple comparison test. White bars indicate no significant difference. B. Black and white regions indicate regions homozygous for 'Habataki' and 'Koshihikari', respectively. ** represents significance at $P<0.01$. The double-sided arrow indicates the candidate region of the locus affecting PPG. 
KHSL-19 were similar to those of 'Koshihikari' (Supplemental Table 2). Therefore, we selected KHSL-19 as a promising line for carrying 'Habataki'-derived gene(s) that may enhance the brown rice appearance quality of plants with a 'Koshihikari' genetic background. By comparing the locations of the substituted chromosomal segments in KHSL-18 and KHSL-19, we determined that the locus affecting appearance quality must be located between RM6767 and the end of chromosome 7 (Fig. 2B).

\section{Mapping the locus on chromosome 7 for grain appearance quality}

To precisely map the putative locus on chromosome 7 , paired line populations were developed through the process shown in Fig. 3. A plant with a heterozygous segment between SSR marker RM6011 and the end of chromosome 7 was identified in the $\mathrm{BC}_{5} \mathrm{~F}_{2}$ population (Supplemental Fig. 2), and its self-progeny (192 plants) were used for the selection of recombinant plants. We selected six plants in which recombination occurred between RM6432 and RM5720. We then self-pollinated each recombinant plant, and identified offspring plants that were homozygous for either the 'Habataki' or the 'Koshihikari' genomic segments in the region of interest. These plants were used as paired line populations (Pair1-1 to Pair1-6) for substitution mapping of the locus in 2010. We found significant differences in the PPGs between the lines in Pair1-2, Pair1-3, and Pair14, but not in Pair1-1, Pair1-5, or Pair1-6 (Fig. 4A). This indicated that the three 'Habataki' substitution lines, Pair1$2 \mathrm{H}$, Pair1-3H, and Pair1-4H, carried the 'Habataki' allele at the locus. This analysis mapped the locus to the chromosomal region flanked by the markers RM1132 and RM8261 (Fig. 4A).

To further narrow down the candidate genomic region of the QTL, nine plants were selected from the progeny (576 individuals) of the same heterozygous parents of the Pair1-3 plants. The selected plants were used to produce nine paired lines (Pair2-1 to Pair2-9), which were analyzed according to the procedure mentioned above. As a result, the target locus was mapped to the chromosomal region flanked by the markers EB_indel-03 and RM21975-2 (Fig. 4B). We then selected eleven plants from the progeny (1755 individuals) of the heterozygote, which had a heterozygous segment between RM6432 and RM3555. These were used to produce eleven paired lines (Pair3-1 to Pair3-11) according to the procedure mentioned above. The analysis of the Pair3 lines clearly indicated that the target locus was located between Tak6166-3 and RM21971 (Fig. 4C). The PPGs obtained for all of the Pair1, Pair2, and Pair3 lines that carried the target locus were similar. The heading dates of all paired lines were similar to that of 'Koshihikari' each year. The average temperatures were $29.4^{\circ} \mathrm{C}$ in $2010,28.2^{\circ} \mathrm{C}$ in 2012 , and $27.0^{\circ} \mathrm{C}$ in 2013. We named the putative locus Apql (Appearance quality of brown rice 1).

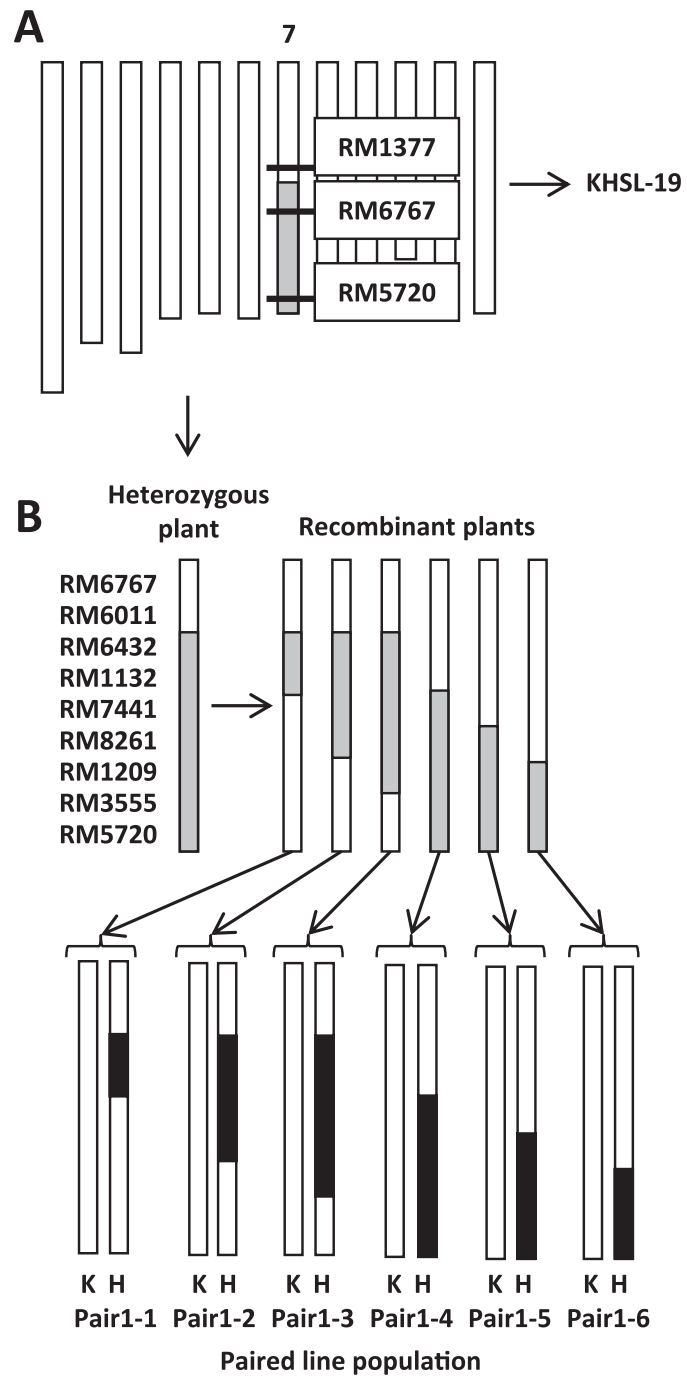

Fig. 3. Flowchart illustrating the development of the paired line populations. Grey, white, and black boxes indicate heterozygous, 'Koshihikari' homozygous, and 'Habataki' homozygous chromosomal regions, respectively. A. The genotype of the plant from which KHSL19 was derived. B. Only chromosome 7 is illustrated. Heterozygous plants, recombinant plants, and paired line populations are shown. $\mathrm{K}$ and $\mathrm{H}$ indicate lines homozygous for 'Koshihikari' and 'Habataki', respectively.

Grain appearance quality and agronomic traits in the Apq1-NIL

The Pair2-8H line (Fig. 4B) showed substitution for the chromosome region flanked by the markers EB_2 to RM3555 (2.8 Mbp). We used Pair2-8H as the Apq $\overline{1}$-NIL and Pair2-8K as the cont-NIL.

The effect of Apq1 under high temperature stress during the ripening period was evaluated in temperature-controlled growth chambers. In 2012 under high temperature conditions, the PPG of the Apq1-NIL (90.0\%) was much higher than those of 'Koshihikari' (44.7\%) and the cont-NIL (47.3\%) (Fig. 5A). Under normal temperature conditions, the PPGs of the Apq1-NIL, 'Koshihikari', and the cont-NIL 


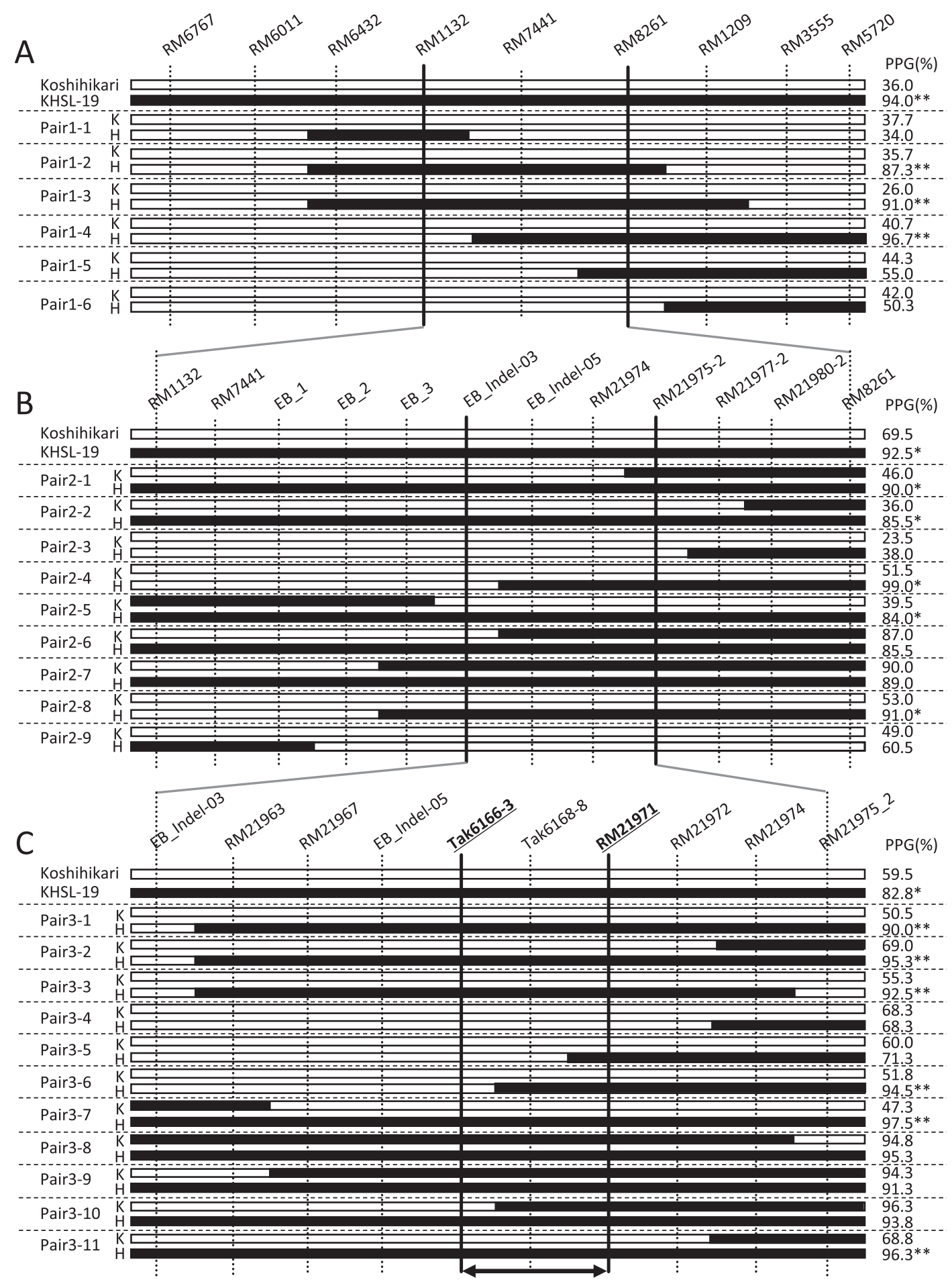

Fig. 4. Mapping of Apql using paired line populations. Black and white regions indicate regions homozygous for 'Habataki' and 'Koshihikari', respectively. The PPG data were obtained in 2010 for Pair1 lines (A), 2012 for Pair2 lines (B), and 2013 for Pair3 lines (C). * and ** represent significance at $P<0.05$ and $P<0.01$, respectively, according to the Student's $t$-test. The double-sided arrow indicates the localized region of Apq1.

were $96.3 \%, 85.3 \%$, and $77.3 \%$, respectively. The results in 2013 showed similar trends as in 2012, though the PPG of the Apq1-NIL in 2013 was lower than in 2012 due to increased proportions of milky-white, white-back, and basalwhite kernels (Fig. 5B). These results indicated that the
Apq1-NIL could produce grains with higher PPG values than those of 'Koshihikari' and the cont-NIL under high temperature stress conditions.

We also compared various agronomic traits between the Apq1-NIL and 'Koshihikari' under field conditions (Table 1). 


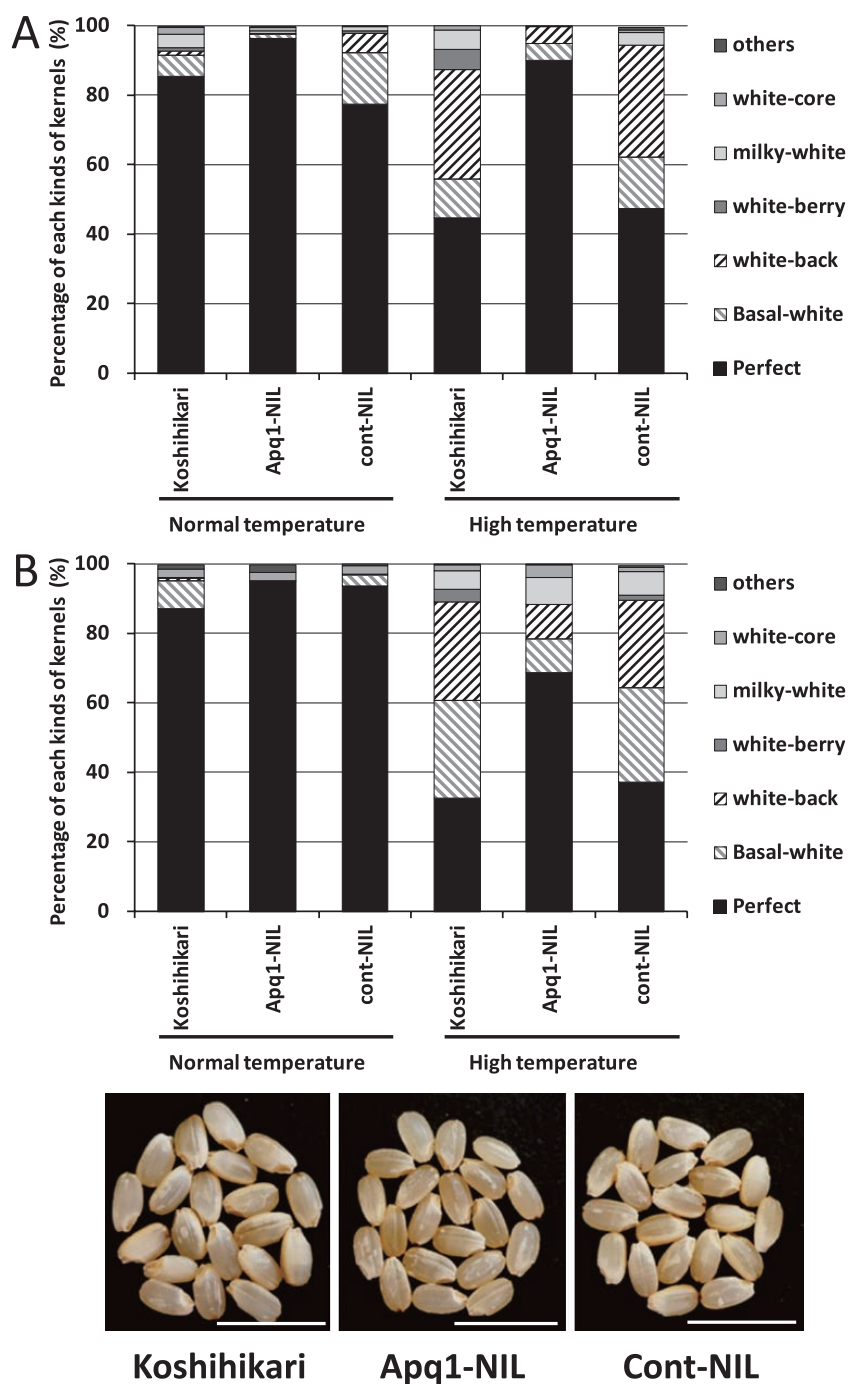

Fig. 5. Grain appearance qualities of Apql-NIL, 'Koshihikari', and cont-NIL plants grown in growth chambers under normal and high temperature conditions in 2012 (A) and 2013 (B). Photos (left, 'Koshihikari'; center, Apq1-NIL; right, cont-NIL) show grains which were produced under high temperature conditions $\left(28.2^{\circ} \mathrm{C}\right.$ average temperature). Scale bar: $1 \mathrm{~cm}$.
The average temperature in 2011 and 2012 were $27.7^{\circ} \mathrm{C}$ and $28.2^{\circ} \mathrm{C}$, respectively. In 2011 there were no significant differences between the two lines regarding heading date, 1,000-grain weight, or any other important trait. In 2012, the culm length and the 1,000-grain weight differed significantly between the Apql-NIL and 'Koshihikari'. The PPG of the Apq1-NIL appeared to be superior to that of 'Koshihikari'.

\section{Discussion}

In the present study, we identified a genomic region on chromosome 7 in 'Habataki' that enhances tolerance to high temperature stress during the ripening period. This tolerance is conferred by the 'Habataki' allele of $A p q 1$ as indicated by clear phenotypic differences in paired lines carrying the 'Habataki' allele (Fig. 4). Several QTLs affecting brown rice quality have previously been reported: He et al. (1999) detected a QTL on chromosome 8; Tan et al. (2000) detected a QTL on chromosome 5; Wan et al. (2005b) identified QTLs on chromosomes 8 and 9; Tabata et al. (2007) found QTLs on chromosomes 1, 2, and 8; Ebitani et al. (2008) detected QTLs on chromosomes 2, 9, 11, and 12; and Kobayashi et al. (2013) identified QTLs on chromosomes 6 and 9. Based on these chromosomal locations, the locus detected in the present study is different from the previously reported QTLs.

Based on PPGs, the appearance quality of brown rice derived from the Apq1-NIL grown at high temperatures in the growth chamber was clearly superior to those of the cont-NIL and 'Koshihikari' in $2013\left(28.2^{\circ} \mathrm{C}\right)$ as well as $2012\left(27.4^{\circ} \mathrm{C}\right)$ (Fig. 5). Moreover, the PPGs of almost all lines harboring the 'Habataki' Apq1 allele were consistently above $85 \%$ in each of the three years, including 2010 , which had a very high average temperature of $29.4^{\circ} \mathrm{C}$ (Fig. 4). Kondo et al. (2006) and Wakamatsu et al. (2007) reported that the PPG decreased when the average temperature rose to more than $27^{\circ} \mathrm{C}$. Moreover, in recent years, the average temperatures in areas where 'Koshihikari' is grown have often exceeded $28^{\circ} \mathrm{C}$. Therefore, the effects of the Apql

Table 1. Comparison of agronomic traits between the Apql-NIL and 'Koshihikari'

\begin{tabular}{|c|c|c|c|c|c|c|c|c|c|c|}
\hline Year & Line/Variety & $\begin{array}{c}\text { Heading } \\
\text { date } \\
(\mathrm{m} / \mathrm{d})\end{array}$ & $\begin{array}{l}\text { Culm } \\
\text { length } \\
(\mathrm{cm})\end{array}$ & $\begin{array}{l}\text { Panicle } \\
\text { length } \\
(\mathrm{cm})\end{array}$ & $\begin{array}{l}\text { Panicle } \\
\text { number } \\
\left(\mathrm{No} . / \mathrm{m}^{2}\right)\end{array}$ & $\begin{array}{c}\text { Lodging } \\
\text { degree }^{a}\end{array}$ & $\begin{array}{l}\text { Yield } \\
(\mathrm{kg} / \mathrm{a})\end{array}$ & $\begin{array}{c}\text { 1000-grain } \\
\text { weight } \\
(\mathrm{g})\end{array}$ & $\begin{array}{c}\text { Percentage } \\
\text { of perfect } \\
\text { grains }(\%)\end{array}$ & $\begin{array}{c}\text { Eating } \\
\text { quality }^{b}\end{array}$ \\
\hline \multirow[t]{3}{*}{2011} & $A p q 1-\mathrm{NIL}$ & $8 / 3$ & 92 & 18.7 & 440 & 3.3 & 56.5 & 22.7 & 88.7 & -0.04 \\
\hline & Koshihikari & $8 / 3$ & 91 & 19.2 & 402 & 1.8 & 59.4 & 23.5 & 83.0 & -0.06 \\
\hline & & n.s. ${ }^{c}$ & n.s. & n.s. & n.s. & n.s. & n.s. & n.s. & n.s. & n.s. \\
\hline \multirow[t]{3}{*}{2012} & Apq1-NIL & $8 / 1$ & 82 & 17.8 & 396 & 0.8 & 57.8 & 23.1 & 91.5 & -0.02 \\
\hline & Koshihikari & $8 / 2$ & 85 & 18.2 & 397 & 1.0 & 57.3 & 23.4 & 73.5 & 0.03 \\
\hline & & n.s. & $*$ & n.s. & n.s. & n.s. & n.s. & $*$ & $* *$ & n.s. \\
\hline
\end{tabular}

${ }^{a}$ Lodging degree was classified into 6 levels ( 0 indicating standing to 5 indicating lodged).

${ }^{b}$ Eating quality shows the aggregate value for overall evaluation and was classified into 9 levels ( 2 indicating excellent to -2 indicating especially bad).

${ }^{c}$ n.s. denotes no significant difference between the Apq1-NIL and 'Koshihikari'. * and $* *$ indicate that the differences between Apq1-NIL and 'Koshihikari' were significant at $P<0.05$ and $P<0.01$, respectively. 
allele evidently are useful.

The chromosomal location of the Apql locus was delimited to a region of $48 \mathrm{~kb}$ between Tak6166-3 and RM21971 (Fig. 4C). In the Rice Annotation Project Database (http:// rapdb.dna.affrc.go.jp/), there are five predicted genes (Os07g0616800, Os07g0616900, Os07g0617000, Os07g0617100, and Os07g0617500) within this region. The Rice Expression Profile Database (http://ricexpro.dna. affrc.go.jp/) provides gene expression profiles from microarray analyses. Data from this database indicate that Os07g0616800 is expressed more highly than the other four genes and that $0 s 07 g 0616800$ is particularly strongly expressed in the ovary and the endosperm at the filling stage. In addition, RNA-seq data from the Rice Annotation Project Database indicate that $O s 07 g 0616800$ is highly expressed in seeds and panicles after flowering. Although linkage analysis allowed us to identify several positional candidate genes, further studies, including a more detailed expression analysis and genetic complementation analysis, will be required to clone the Apq1 gene.

All agronomic traits of the Apq1-NIL were similar to those of 'Koshihikari' (Table 1). The culm length and 1,000-grain weight differed slightly between the Apq1-NIL and 'Koshihikari' in 2012; however, these small differences would cause no practical difficulties in a commercially grown crop. The appearance quality of brown rice is generally affected by heading date because of changes in temperature during the ripening period (Hori et al. 2012). Ebitani et al. (2008) reported that high PPG lines tend to have lighter 1,000-grain weights. On the other hand, the heading date and 1,000-grain weight of the Apq1-NIL were similar to those of 'Koshihikari'. In addition, the Apq1-NIL shows no disadvantages in yield and eating quality compared with 'Koshihikari'. Therefore, we conclude that the Apq1 allele from 'Habataki' improves grain quality without affecting other important agronomic traits.

\section{Acknowledgments}

This work was supported by grants from the Ministry of Agriculture, Forestry and Fisheries of Japan (Development of mitigation and adaptation techniques to global warming in the sectors of agriculture, forestry, and fisheries, 1106). We thank Dr. Tatsuro Hirose for his comments on this manuscript.

\section{Literature Cited}

Ebitani, T., Y. Yamamoto, M. Yano and M. Funane (2008) Identification of quantitative trait loci for grain appearance using chromosome segment substitution lines in rice. Breed. Res. 10: 91-99.

Ebitani, T., N. Hayashi, M. Omoteno, H. Ozaki, M. Yano, M. Morikawa and Y.Fukuta (2011) Characterization of Pi13, a blast resistance gene that maps to chromosome 6 in indica rice (Oryza sativa L. variety, Kasalath). Breed. Sci. 61: 251-259.

Ebitani, T., Y. Iyama, T. Yamaguchi, H. Ozaki and Y.Kidani (2013) Attempt to improve of lodeging resistance, blast resistance and ripening ability in high temperature stress of the Japanese elite cultivar, "Koshihikari" retaining the genetic background. Bull. Agr. Res. Inst., Toyama Pref. Agr., For. Fish. Res. Ctr. 5: 1-6.

Fukuoka, S., N.Saka, H.Koga, K. Ono, T.Shimizu, K. Ebana, N.Hayashi, A.Takahashi, H. Hirochika, K. Okuno et al. (2009) Loss of function of a proline-containing protein confers durable disease resistance in rice. Science 325: 998-1001.

Harushima, Y., M. Yano, A. Shomura, M. Sato, T. Shimano, Y.Kuboki, T. Yamamoto, S.Y.Lin, B.A.Antonio, A.Parco et al. (1998) A high-density rice genetic linkage map with 2275 markers using a single $\mathrm{F}_{2}$ population. Genetics 148: 479-494.

He, P., S.G.Li, Q. Qian, Y.Q.Ma, J.Z.Li, W.M.Wang, Y.Chen and L.H.Zhu (1999) Genetic analysis of rice grain quality. Theor. Appl. Genet. 98: 502-508.

Hori, K., T.Kataoka, K. Miura, M.Yamaguchi, N.Saka, T. Nakahara, Y. Sunohara, K. Ebana and M. Yano (2012) Variation in heading date conceals quantitative trait loci for other traits of importance in breeding selection of rice. Breed. Sci. 62: 223-234.

International Rice Genome Sequencing Project (2005) The map-based sequence of the rice genome. Nature 436: 793-800.

Ishizaki, K., T. Hoshi, S. Abe, Y. Sasaki, K. Kobayashi, H. Kasaneyama, T. Matsui and S.Azuma (2005) Breeding of blast resistant isogenic lines in rice variety "Koshihikari" and evaluation of their characters. Breed. Sci. 55: 371-377.

Kobayashi,A., Y.Koga, H.Uchiyamada, H.Horiuchi, K. Miura, K. Okuno, Y. Fujita, Y.Uehara, S. Ishizaka, M.Nakagahra et al. (1990) Breeding a new rice variety "Habataki". Bull. Hokuriku Natl. Agric. Exp. 32: 65-84.

Kobayashi,A., J.Sonoda, K. Sugimoto, M.Kondo, N. Iwasawa, T.Hayashi, K. Tomita, M. Yano and T.Shimizu (2013) Detection and verification of QTLs associated with heat-induced quality decline of rice (Oryza sativa L.) using recombinant inbred lines and near-isogenic lines. Breed. Sci. 63: 339-346.

Kojima, Y., T. Ebitani, Y. Yamamoto and T. Nagamine (2004) Development and utilization of isogenic lines 'Koshihikari' Toyama BL. In: Kawasaki, S. (ed.) 'Rice Blast: Interaction with Rice and Control, Kluwer Academic Publishers, Dordrecht, Netherlands, pp. 209214.

Kondo,M., S.Morita, K.Nagata, Y.Koyama, N.Ueno, J.Hosoi, Y. Ishida, T. Yamakawa, Y. Nakayama, Y.Yoshioka et al. (2006) Effects of air temperature during ripening and grain protein contents on grain chalkiness in rice. Jpn. J. Crop Sci. 75 (Extra issue 2): 14-15.

Kurata, N., Y.Nagamura, K. Yamamoto, Y.Harushima, N. Sue, J.Wu, B.A.Antonio, A. Shomura, T. Shimizu, S.Y.Lin et al. (1994) A 300 kilobase interval genetic map of rice including 883 expressed sequences. Nat. Genet. 8: 365-372.

McCouch, S.R., L. Teytelman, Y.Xu, K.B.Lobos, K. Clare, M. Walton, B. Fu, R. Maghirang, Z. Li, Y.Xing et al. (2002) Development and mapping of 2240 new SSR markers for rice (Oryza sativa L.). DNA Res. 9: 199-207.

Murray, M.G. and W.F. Thompson (1980) Rapid isolation of high molecular weight plant DNA. Nucleic Acids Res. 8: 4321-4325.

Tabata, M., H. Hirabayashi, Y. Takeuchi, I. Ando, Y. Iida and R. Ohsawa (2007) Mapping of quantitative trait loci for the occurrence of white-back kernels associated with high temperatures during the ripening period of rice (Oryza sativa L.) Breed. Sci. 57: 47-52.

Takeuchi, Y., T. Ebitani, T. Yamamoto, H. Sato, H. Ohta, H. Hirabayashi, H. Kato, I.Ando, H. Nemoto, T. Imbe et al. (2006) Development of isogenic lines of rice cultivar Koshihikari with early and late heading by marker-assisted selection. Breed. Sci. 56: 405-413. 
Tan, Y.F., Y.Z. Xing, J.X. Li, S.B. Yu, C.G. Xu and Q. Zhang (2000) Genetic bases of appearance quality of rice grains in Shanyou 63, an elite rice hybrid. Theor. Appl. Genet. 101: 823-829.

Wakamatsu, K., O. Sasaki, I. Uezono and A. Tanaka (2007) Effects of high air temperature during the ripening period on the grain quality of rice in warm regions of Japan. Jpn. J. Crop Sci. 76: 71-78.

Wan, Z-X., S. Sakaguchi, Y. Oka, N. Kitazawa and Y.Minobe (2005a) Breeding of semi-dwarf Koshihikari by using genome breeding method. Breed. Res. 7 (Suppl. 1, 2): 217.

Wan, X.Y., J.M.Wan, J.F.Weng, L.Jiang, J.C.Bi, C.M.Wang and H.Q.Zhai (2005b) Stability of QTLs for rice grain dimension and endosperm chalkiness characteristics across eight environments. Theor. Appl. Genet. 110: 1334-1346.

Yamamoto, T., N. Horisue and R. Ikeda (1996) Rice breeding manual. Yokendo Co. Ltd., Tokyo, Japan, pp. 3-156. 